\title{
EXPOSING NEW COMPONENTS OF THE X-RAY BACK- GROUND WITH MULTI-WAVELENGTH SKY SURVEYS
}

\author{
E.C. MORAN \\ Lawrence Livermore National Laboratory
}

\section{Introduction}

The evolving class of objects responsible for the majority of the cosmic $\mathrm{X}$-ray background (XRB) remains at large nearly three and a half decades after the discovery of the XRB. Surveys of sources selected on the basis of their X-ray properties alone provide an unbiased picture of the X-ray sky, but to date they have not been ideal for the discovery of rare types of $\mathrm{X}$-ray sources at faint fluxes: large-area X-ray surveys have been restricted to bright sources, while deep X-ray surveys have been limited to very small patches of sky. X-ray selection coupled with another selection criterion, e.g., a radio or infrared detection, complements "pure" X-ray surveys by (1) permitting the exploration of large areas of sky to faint flux limits for types of extragalactic X-ray sources not well represented in other surveys, and (2) assisting the location of the optical counterparts of these X-ray sources. Using this approach, I have searched for new components of the XRB among the faintest X-ray sources detected by the Einstein Observatory.

\section{The Einstein Two-Sigma Catalog}

For certain applications, the limiting signal-to-noise threshold for sources in an astronomical catalog may be considerably lower than commonly accepted values (i.e., $4 \sigma$ or $5 \sigma$ ), provided that one can determine the statistical reliability of the catalog. To facilitate the study of X-ray sources fainter than those contained in the Einstein Medium Sensitivity Survey (EMSS; Gioia et al. 1990), we have constructed a new catalog of 46,186 sources and fluctuations exceeding $2 \sigma$ significance in 2520 high-latitude Einstein IPC images. We have employed various tests to validate our source-search 
algorithm for both high- and low-significance sources, and to identify and remove the small number of spurious sources induced by our detection procedure. Based on the known properties of the Einstein optics, the background characteristics of the IPC, and the measured X-ray $\log N-\log S$ relation, we have modeled the number of real sources expected in the cata$\log$ in order to evaluate its statistical properties below $4 \sigma$ significance. Our model suggests that $\sim 28 \%$, or $\sim 13,000$, of the sources in the Two-Sigma Catalog are real celestial X-ray sources. This is an increase of $\sim 9100$ over the number found in previous analyses of the same IPC images. Full details of the manufacture and evaluation of the Two-Sigma Catalog are described in Moran et al. (1996).

\section{New Components of the Cosmic X-ray Background}

The primary motivation for assembling large samples of faint X-ray sources is to search for possible new components of the X-ray background. As a means of selecting real celestial X-ray sources in the Two-Sigma Catalog for further study, we have applied astronomical catalogs at other wavelengths as filters. The cross-correlation of these catalogs with the Two-Sigma Catalog produces samples of hundreds of faint X-ray sources that are reliable at the $90 \%$ level. The specific filters we have used are catalogs from surveys of the radio and infrared sky.

Optical spectroscopy of 77 unidentified radio- and IR-selected TwoSigma Catalog sources has turned up several surprises, illustrating the merits of selecting X-ray sources by a variety of methods. In addition to the types of objects one would expect to find in these samples, we have discovered high-redshift quasars (one at $z=4.30$ ), which are absent in the EMSS, $\mathrm{X}$-ray-luminous radio-loud elliptical galaxies with optical spectra devoid of emission lines, and infrared-bright AGNs with optical spectra dominated by starburst galaxy features (Moran et al. 1996). Follow-up X-ray observations are being carried out with ROSAT and ASCA to clarify the nature of these objects and to determine if any of them represents a previously unrecognized component of the X-ray background.

\section{References}

Gioia, I. M., et al. 1990, Astrophys.J.Supp. 72, 567.

Moran, E. C., Helfand, D. J., Becker, R. H., \& White, R. L. 1996, Astrophys.J. 461, 127. 